\title{
Comparative effects of sex hormone deprivation on the brain of insulin- resistant rats
}

\author{
Jirapas Sripetchwandee1,2, Hiranya Pintana1, Piangkwan Sa-nguanmoo1, Chiraphat Boonnag1, \\ Wasana Pratchayasakul1,2, Nipon Chattipakorn ${ }^{1,2}$ and Siriporn C Chattipakorn ${ }^{1,3}$
}

${ }^{1}$ Neurophysiology Unit, Cardiac Electrophysiology Research and Training Center, Faculty of Medicine, Chiang Mai University, Chiang Mai, Thailand 2Department of Physiology, Faculty of Medicine, Chiang Mai University, Chiang Mai, Thailand

3Department of Oral Biology and Diagnostic Sciences, Faculty of Dentistry, Chiang Mai University, Chiang Mai, Thailand

Correspondence should be addressed to S C Chattipakorn: siriporn.c@cmu.ac.th

\begin{abstract}
Obese-insulin resistance following chronic high-fat diet consumption led to cognitive decline through several mechanisms. Moreover, sex hormone deprivation, including estrogen and testosterone, could be a causative factor in inducing cognitive decline. However, comparative studies on the effects of hormone deprivation on the brain are still lacking. Adult Wistar rats from both genders were operated upon (sham operations or orchiectomies/ovariectomies) and given a normal diet or high-fat diet for 4, 8 and 12 weeks. Blood was collected to determine the metabolic parameters. At the end of the experiments, rats were decapitated and their brains were collected to determine brain mitochondrial function, brain oxidative stress, hippocampal plasticity, insulin-induced long-term depression, dendritic spine density and cognition. We found that male and female rats fed a high-fat diet developed obese-insulin resistance by week 8 and brain defects via elevated brain oxidative stress, brain mitochondrial dysfunction, impaired insulin-induced long-term depression, hippocampal dysplasticity, reduced dendritic spine density and cognitive decline by week 12 . In normal diet-fed rats, estrogen deprivation, not testosterone deprivation, induced obese-insulin resistance, oxidative stress, brain mitochondrial dysfunction, impaired insulin-induced long-term depression, hippocampal dysplasticity and reduced dendritic spine density. In high-fat-diet-fed rats, estrogen deprivation, not testosterone deprivation, accelerated and aggravated obese-insulin resistance and brain defects at week 8 . In conclusion, estrogen deprivation aggravates brain dysfunction more than testosterone deprivation through increased oxidative stress, brain mitochondrial dysfunction, impaired insulin-induced long-term depression and dendritic spine reduction. These findings may explain clinical reports which show more severe cognitive decline in aging females than males with obeseinsulin resistance.
\end{abstract}

\author{
Key Words \\ - cognitive impairment \\ - obesity \\ - sex hormone \\ - high-fat diet consumption \\ - insulin resistance \\ - oxidative stress
}




\section{Introduction}

Insulin resistance, a chronic pathological condition, can lead to several complications such as type 2 diabetes mellitus (Taylor 2012), heart diseases (Ginsberg 2002) and cognitive decline (Watts et al. 2013). We have reported previously that chronic consumption of high-fat diet (HFD) could not only induce obese condition and peripheral insulin resistance but also lead to cognitive impairment. We proposed that these impairments were caused as a result of several mechanisms including brain insulin resistance, brain oxidative stress, impaired mitochondrial function in brain, changes in synaptic plasticity of hippocampus and loss of dendritic spine (Pratchayasakul et al. 2011, 2015, Pipatpiboon et al. 2012, 2013, Pintana et al. 2013, 2015a). In addition, a recent study demonstrated that impairment of brain redox homeostasis occurred under obese-insulin-resistant condition (Maciejczyk et al. 2018).

Despite an important role in promoting secondary sexual characteristics in males (Schiavi \& White 1976), testosterone also plays a significant role in cognition (Hogervorst et al. 2001, Kenny et al. 2004, Pintana et al. 2015a). It has been reported that testosterone deficiency, caused by orchiectomy, resulted in insulin resistance in periphery (Grossmann et al. 2008, Xia et al. 2013), subsequently resulting in impaired learning and memory loss (Sakata et al. 2000, Naghdi et al. 2005, Sandstrom et al. 2006, Pintana et al. 2015b). Consistent with the findings from testosterone deprivation, a number of studies also reported that ovariectomy-induced estrogen deficiency also led to peripheral insulin resistance and cognitive decline (Daniel et al. 1997, Luine et al. 1998, Pratchayasakul et al. 2015). Taken together, these findings suggested that sex hormones are required to preserve cognitive function for each gender. Although it has already been reported that the deficiency of these hormones causes cognitive impairment, we propose that it would be interesting to explore whether both hormones have equal effects on the brain under conditions of deprivation in both the presence and absence of obese-insulin-resistant conditions. Thus, we tested the hypothesis that the deprivation of these sex hormones, testosterone and estrogen, had equal effects in inducing the brain dysfunction of rats received with a normal diet (ND) or a HFD.

\section{Materials and methods}

One-hundred and forty-four Wistar rats of both male and female gender ( $n=72 /$ gender, 180-200g) were obtained from the National Animal Center, Salaya Campus, Mahidol University, Bangkok, Thailand, for use in the experiments. All experiments were performed in line with the protocol approved by the Faculty of Medicine, Chiang Mai University Institutional Animal Care and Use Committee, in compliance with $\mathrm{NIH}$ guidelines (28/2556 and 6/2557). Rats were housed in environmentally controlled conditions $\left(25 \pm 0.5^{\circ} \mathrm{C}, 12\right.$-h light/darkness cycle) with free access to drinking water. Then, rats from each gender were randomly separated into two groups ( $n=36 /$ group) including sham-operated or orchiectomized/ovariectomized rats. One week after surgery, rats in each group were subsequently divided into two groups ( $n=18 /$ group) to be given with a ND (Supplementary Table 1, see section on supplementary data given at the end of this article) $(19.77 \% \mathrm{E}$ fat without dates) or a high-fat diet (Supplementary Table 2) $(59.28 \%$ E fat, HFD) for 4, 8 and 12 weeks ( $n=6 /$ time point). Food was provided ad libitum (Sakata et al. 2000). At each time point, animals from each gender were separated into four groups, such as ND with sham operation, ND with orchiectomy/ovariectomy, HFD with sham operation and HFD with orchiectomy/ovariectomy. The body weight of animal was recorded once a week, while food intake was also recorded daily. Blood sampling from a tail vein was performed at the end of each time period including 4 , 8 and 12 weeks for metabolic analyses including serum oxidative stress, plasma levels of estrogen, testosterone, insulin and glucose, and lipid profiles including total cholesterol (TC), low- and high-density lipoprotein (LDL and HDL) and triglyceride (TG). Regarding the collection of blood samples, NaF-coated tubes were used for plasma glucose determination, whereas EDTA-coated tubes were used for other metabolic parameters. In addition, clotted blood was used for determining serum malondialdehyde (MDA) levels. An oral glucose tolerance test (OGTT) was determined at 4, 8 and 12 weeks in the schedule of dietary consumption, as previously reported (Pratchayasakul et al. 2015, Pintana et al. 2016). After the 12-week period, all rats were anesthetized using isoflurane prior to the decapitation. After that, brain was immediately removed and brain slices were prepared for the extracellular recordings such as insulin-induced long-term depression (LTD) and synaptic long-term potentiation (LTP), and 
brain mitochondrial function. Brain samples were also kept in $-80^{\circ} \mathrm{C}$ for determining brain oxidative stress and dendritic spine density.

\section{Ovariectomy protocol}

In carrying out of the ovariectomy, female rats were intraperitoneally injected with $0.15-\mathrm{mL} / \mathrm{kg}$ Xylazine (LBS Laboratories, Bangkok, Thailand) and $50 \mathrm{mg} / \mathrm{kg}$ Zoletil (Virbac Laboratories, Carros, France). Bilateral ovariectomy was conducted by a section at the dorsal midline, the ovaries were thoroughly removed and the incision was consequently closed (Pratchayasakul et al. 2015).

\section{Orchiectomy protocol}

Orchiectomy was conducted using a method as shown in previous reports (Pintana et al. 2015a, 2016). Male rats were anesthetized and the bilateral scrotal approach technique was used. After incision at a tip of the scrotum, tunica albuginea was cut. Then, the vas deferens and blood vessels were then ligated prior to removing the testes. Finally, the other tissues were kept in the sac before closing the incised skin.

\section{The determination of metabolic parameters by chemical analysis}

Plasma levels of TC and glucose were evaluated using colorimetric assay kits (Biotech, Bangkok, Thailand), while plasma levels of insulin were detected by an ELISA kit (Linco Research). The HOMA (Homeostasis Model Assessment) was required to evaluate the severity of insulin resistance. The higher the HOMA index was taken as indicating the severity of insulin resistance at periphery (Pipatpiboon et al. 2012, Pintana et al. 2015b, Pratchayasakul et al. 2015).

With regard to the determination of sex hormone concentration, circulating levels of testosterone were determined by an electrochemiluminescence immunoassay technique (Roche Diagnostic), whereas circulating estrogen levels were detected by an enzymatic immunoassay kit (Cayman Chemical).

\section{The oral glucose tolerance test}

After 4, 8, and 12 weeks of the dietary consumption, rats were fasted for at least $12 \mathrm{~h}$ before undergoing the OGTT. Glucose was then given by gavage feeding at a concentration of $2 \mathrm{~g} / \mathrm{kg}$ body weight. Blood sampling was done immediately after feeding for baseline and at a specific time including 15, 30, 60 and $120 \mathrm{~min}$ after loading of glucose. The plasma glucose level was determined with a commercial kit (Biotech, Bangkok, Thailand) and reported as a plasma glucose area under the curve $\left(\mathrm{AUC}_{\mathrm{g}}\right)$.

\section{Determination of brain mitochondrial function}

Preparation of brain mitochondria was done in accordance with a previous report (Sripetchwandee et al. 2013). Brain mitochondrial reactive oxygen species (ROS) production was examined by a dichlorohydro-fluorescein diacetate dye (Pipatpiboon et al. 2012, Sripetchwandee et al. 2013) and reported as the \% change in brain mitochondrial ROS production. Brain mitochondrial membrane potential change was determined by using a fluorescent JC-1 dye (Pipatpiboon et al. 2012, Sripetchwandee et al. 2013) and demonstrated as the increase in \% change of brain mitochondrial membrane potential. Lastly, a kinetic change in the absorbance of mitochondrial suspensions for 30 minutes was used to determine brain mitochondrial swelling (Pipatpiboon et al. 2012, Sripetchwandee et al. 2013).

\section{Serum and brain oxidative stress assay using MDA detection}

Serum and brain levels of MDA were investigated by a high-liquid performance chromatography-based analysis (Pratchayasakul et al. 2015, Pintana et al. 2016). Briefly, the $\mathrm{H}_{3} \mathrm{PO}_{4}, 10 \%$ trichloro acetic acid (TCA) containing butylated hydroxytoluene (BHT) and thiobarbituric acid (TBA) solution were added into either serum or homogenized brain tissues to produce the TBA-reactive substances (TBARs). After that, the TBAR levels from serum and brain were calculated using a standard curve and demonstrated as the MDA concentrations ( $\mu \mathrm{M}$ and $\mu \mathrm{mol} / \mathrm{g}$ for serum and brain, respectively).

\section{Electrophysiological recording for LTP and LTD}

After preparing the hippocampal slices (Chattipakorn \& McMahon 2002), a field excitatory postsynaptic potentials (fEPSPs) was recorded as per a previous report (Pratchayasakul et al. 2015). For investigating the LTD, brain slices were perfused for $10 \mathrm{~min}$ with artificial cerebrospinal fluid (aCSF) and recorded fEPSPs as baseline. Then, the induction of LTD with insulin was assessed by the perfusion with aCSF with 500-nM insulin (Humulin R, Eli Lilly, Giessen, Germany) for $10 \mathrm{~min}$ after baseline. 
Following this the perfusion with aCSF without insulin was conducted for a further $50 \mathrm{~min}$. A \% decrease of fEPSPs represented insulin-induced LTD. In accordance with the LTP protocol, a high-frequency stimulation (HFS: 4 trains at $100 \mathrm{~Hz}$ with $0.5 \mathrm{~s}$ of duration, with a time interval of 20 s) was used to initiate the LTP induction. The fEPSPs readings were recorded at least $40 \mathrm{~min}$ after HFS. The \% increase of fEPSPs after HFS for at least 30 min indicated a successful LTP protocol.

\section{Golgi staining technique for determining dendritic spine density}

Brain samples were rinsed and immersed in the Rapid Golgi Stain Kit's PK401 solution (FD Neurotechnologies Inc., Ellicott City, MD, USA) for Golgi impregnation (Pratchayasakul et al. 2015, Pintana et al. 2016). Branches of the dendritic spines were identified with a $60 \times$ oilimmersion objective lens of an inverted microscope (IX-81; Olympus). Total number of dendritic spines was calculated and the dendritic length was measured with the Xcellence imaging software (Olympus) and spine density was indicated as the total numbers of spine/10-20 $\mu \mathrm{m}$ of dendritic length.

\section{Determination of cognitive function using Morris water maze test}

Protocol for Morris water maze was adjusted from a previous report (Vorhees \& Williams 2006). The test consists of an acquisition phase using a hidden platform and a probe trial test by a removal of the platform from a pool (Pintana et al. 2012). Rats were trained to find a platform during the acquisition phase and underwent four trials/day for 5 days with a resting time for $15 \mathrm{~s}$ before next trial. Rats were given $120 \mathrm{~s}$ to find the platform for each trial, and recorded the time to reach the platform. The probe test was carried out by the platform being removed from the pool, and the time spent in the target quadrant was recorded. Both parameters were analyzed by Smart 3.0 software (Panlab, Harvard Apparatus, Barcelona, Spain).

\section{Statistical analysis}

Data were presented as mean \pm standard deviation (s.D.). The differences between groups were analyzed using the unpaired Student's $t$ test. $P$ Value $<0.05$ was considered as a statistical significance. Moreover, Pearson's correlation analysis was used to determine the relationship among the assessed parameters.

\begin{tabular}{|lr} 
https://joe.bioscientifica.com & (c) 2019 Society for Endocrinology \\
https://doi.org/10.1530/JOE-18-0552 & Published by Bioscientifica Ltd. \\
Printed in Great Britain
\end{tabular}




\section{Results}

\section{Effects of sex hormone deprivation on metabolic parameters in ND-fed rats}

Testosterone deprivation was confirmed by a reduction of testosterone in orchiectomized rats, compared with sham-operated male rats (Table 1 ). In addition, ovariectomy also reduced the estrogen level in ovariectomized rats when compared with shamoperated female rats (Table 1). Testosterone deprivation caused by orchiectomy led to a significant reduction in body weight, food intake and the visceral fats of male rats that received a ND for 4,8 and 12 weeks (Table 2). However, testosterone deprivation did not cause peripheral insulin resistance in these rats (Table 2). In contrast, estrogen deprivation was associated with the elevation of body weight, visceral fats, plasma insulin, HOMA index and plasma $\mathrm{AUC}_{\mathrm{g}}$ in both 8- and 12-week ND-fed female rats when compared with sham-operated groups, indicating the development of peripheral insulin resistance from estrogen deprivation (Table 2). In ND-fed rats with a sham operation in both genders, male rats had higher levels of body weight, food intake, visceral fat, plasma insulin and HOMA index, while the level of TC and LDL was lower than that found in female rats (Table 2). Taken together, these findings suggested that estrogen might be a key player in the development of peripheral insulin resistance.

\section{Effects of HFD consumption at 4, 8 and 12 weeks on metabolic parameters in rats from both genders}

Male rats fed on a HFD for 4 weeks developed obesity as indicated by the significant increase in body weight and visceral fat, compared with ND-fed rats (Table 3). In addition, peripheral insulin resistance was found to have developed after 8 weeks of HFD consumption as indicated by an increase in plasma insulin, HOMA index and plasma $\mathrm{AUC}_{\mathrm{g}}$ (Table 3). Consistent with the findings in male rats, female rats on a HFD for 4 weeks also had increased body weight and visceral fat, and these rats developed peripheral insulin resistance after 8 weeks of HFD consumption (Table 3). These findings indicate that consumption of a HFD for 8 weeks caused peripheral insulin resistance to a similar extent in both genders when their sex hormones were intact.

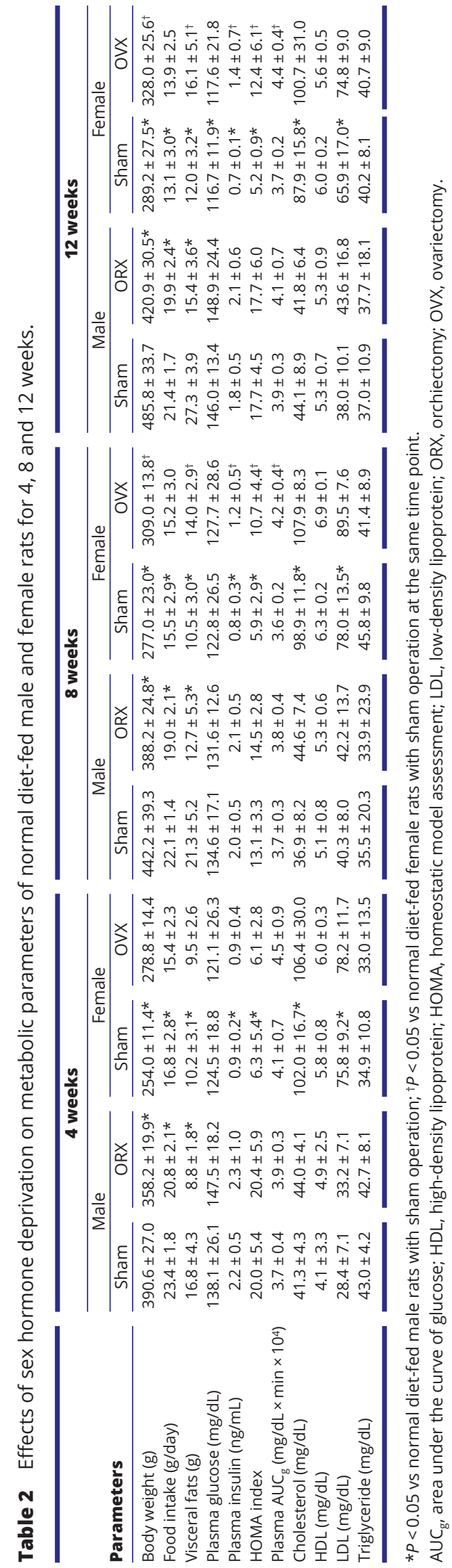



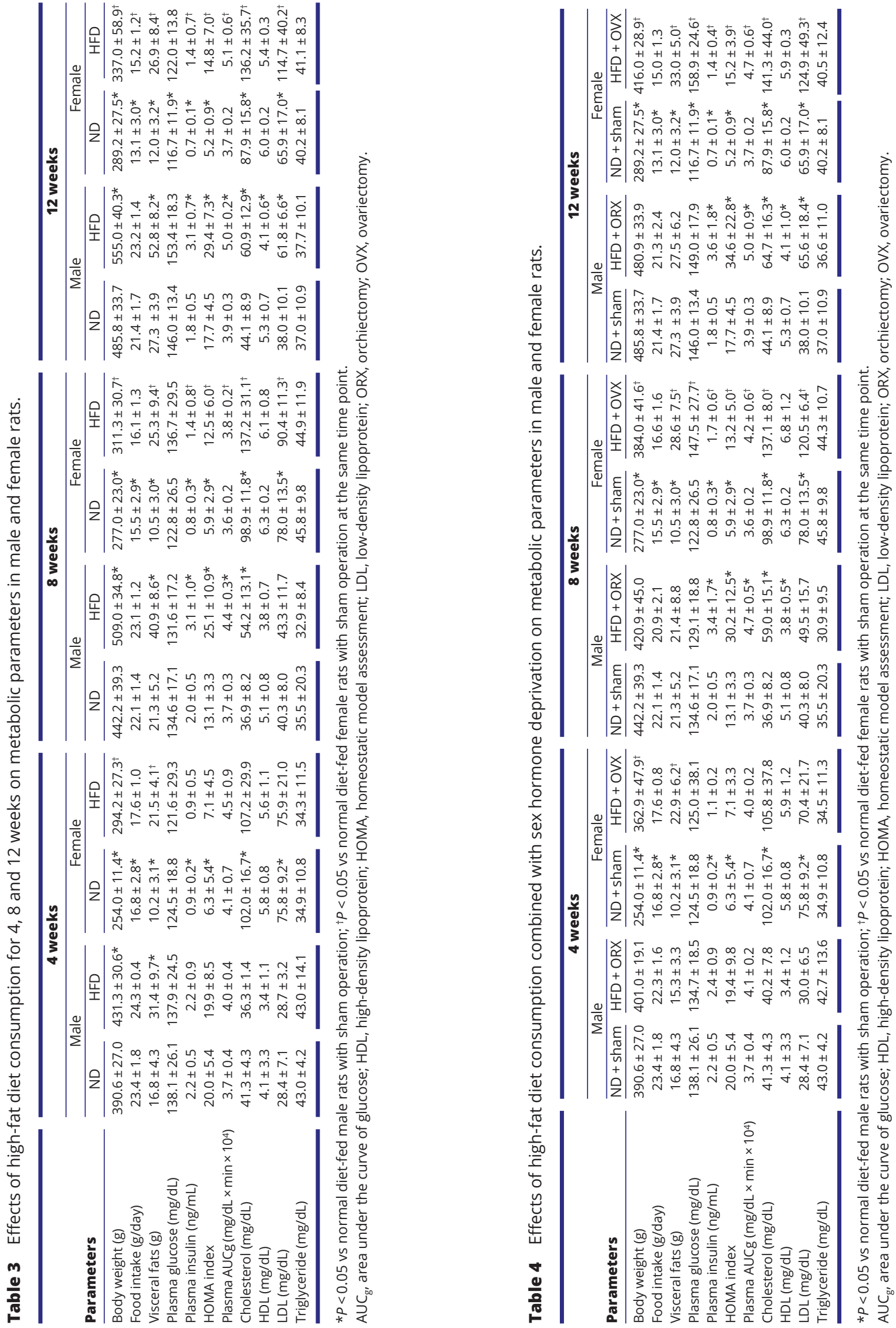

https://joe.bioscientifica.com 

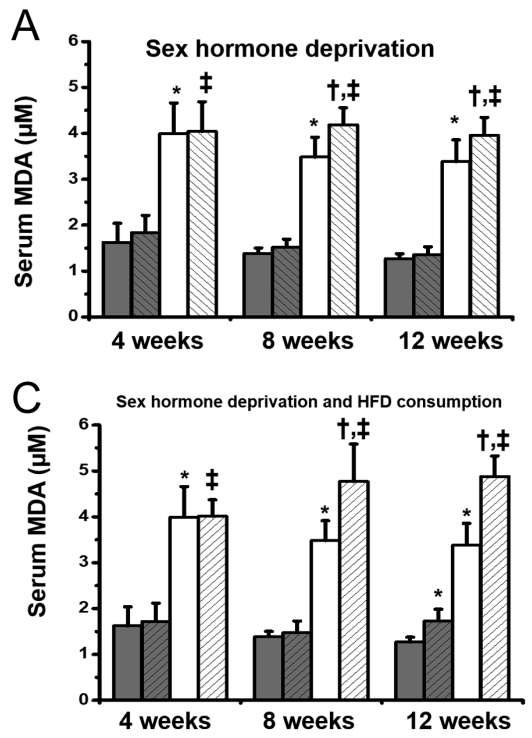

Effects of HFD consumption combined with sex hormone deprivation on metabolic parameters in rats

In male rats, consumption of a HFD for 4 weeks combined with testosterone deprivation did not alter any metabolic parameters when compared with ND-fed rats (Table 4). Peripheral insulin resistance in these rats was observed after 8 weeks of HFD consumption as demonstrated by the increase of plasma insulin, plasma $\mathrm{AUC}_{\mathrm{g}}$ and HOMA index (Table 4). In contrast, female ovariectomized rats have elevated body weight and visceral fats after 4 weeks of HFD consumption (Table 4), and peripheral insulin resistance was observed after 8 weeks of HFD feeding in these rats compared with ND-fed rats with a sham operation (Table 4). These findings indicate that sex hormone deprivation did not accelerate the development of peripheral insulin resistance in both male and female obese rats.

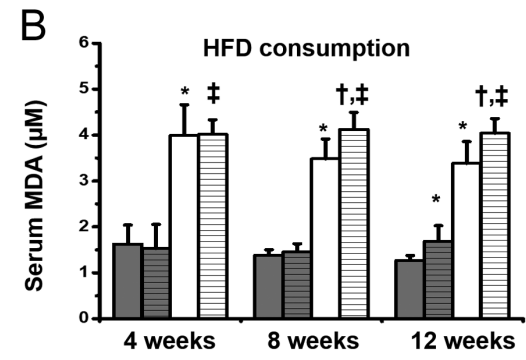

\section{Figure 1}

Effects of sex hormone deprivation: (A) HFD consumption; (B) sex hormone deprivation combined with HFD consumption; (C) the resulting induction of serum oxidative stress marker (MDA). $X$-Axis represents time course of dietary consumption at 4,8 and 12 weeks. $\star P<0.05$ vs normal diet-fed male rats with sham operation, ${ }^{\dagger} P<0.05$ vs normal diet-fed female rats with sham operation and $\neq P<0.05$ vs male gender with same group. HFD, high-fat diet; MDA, malondialdehyde.

\section{Effects of sex hormone deprivation, HFD consumption} and their combined impact on oxidative stress status

Testosterone deprivation alone did not alter the oxidative stress status as shown by no alteration in serum and brain MDA levels compared with ND-fed rats which had undergone a sham operation (Fig. 1). However, HFD consumption for 12 weeks significantly increased the MDA level in both sham-operated and orchiectomized rats (Fig. $1 \mathrm{~B}$ and $\mathrm{C}$ ).

Compared with male rats, estrogen deprivation, HFD consumption and a combination of these conditions caused increased systemic oxidative stress by increasing the serum MDA level of rats which received 8 and 12 weeks of both dietary consumptions, when compared with ND-fed rats which had undergone a sham operation (Fig. 1A, B and C). In addition, levels
A
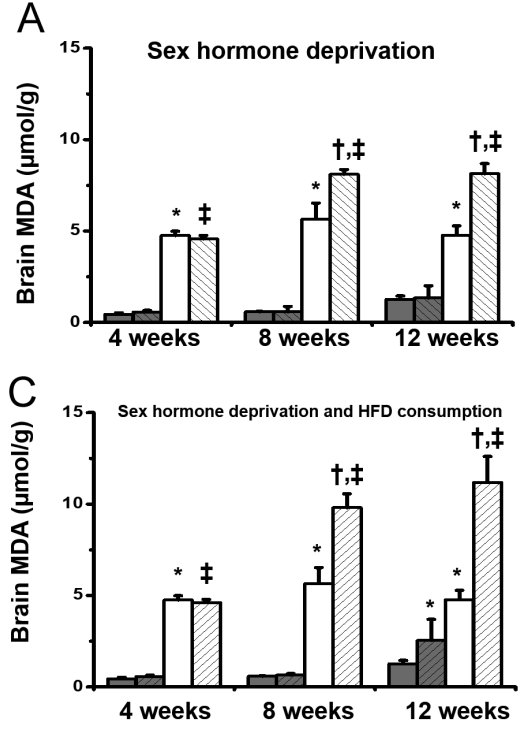

B
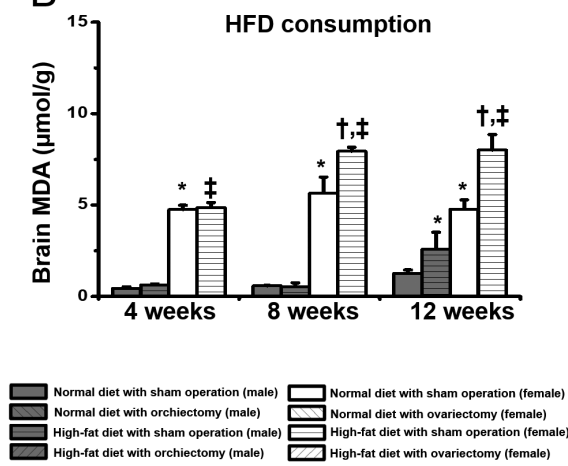

Figure 2

Effects of sex hormone deprivation: (A) HFD consumption; (B) sex hormone deprivation combined with HFD consumption; (C) on the induction of brain oxidative stress marker (MDA). $X$-Axis represents time course of dietary consumption including 4, 8 and 12 weeks. ${ }^{\star} P<0.05$ vs normal diet-fed male rats with sham operation, ${ }^{\dagger} P<0.05$ vs normal diet-fed female rats with sham operation and $¥ P<0.05$ vs male gender with same group. HFD, high-fat diet; MDA, malondialdehyde. 
A

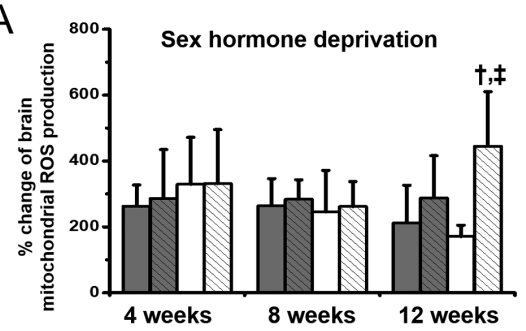

C

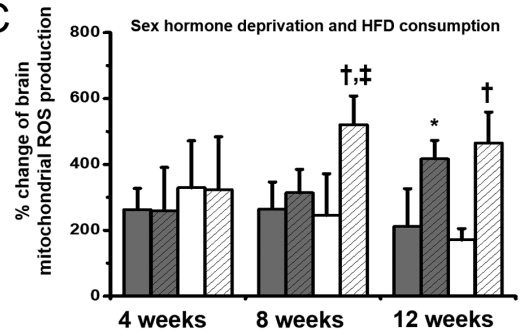

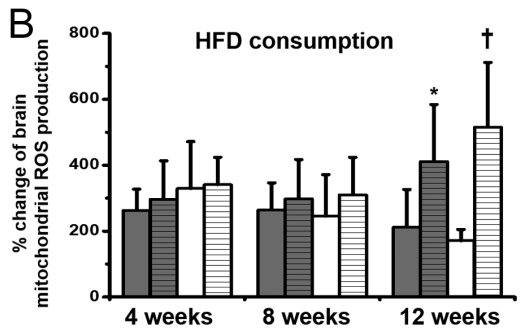

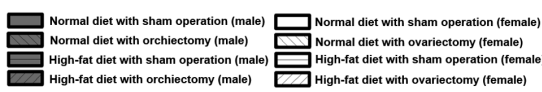

Figure 3

Effects of sex hormone deprivation: (A) HFD consumption; (B) sex hormone deprivation combined with HFD consumption; (C) on the \% change of brain mitochondrial ROS production. $X$-Axis represents time course of dietary consumption at 4,8 and 12 weeks. $* P<0.05$ vs normal diet-fed male rats with sham operation, ${ }^{t} P<0.05$ vs normal diet-fed female rats with sham operation and $¥ P<0.05$ vs male gender with same group. HFD, high-fat diet; ROS, reactive oxygen species. of brain oxidative stress were consistent with that observed in the peripheral samples (Fig. 2A, B and C). However, both serum and brain MDA levels in female rats were higher than in male. These findings indicate that females are more vulnerable to the development of a severity in oxidative stress than males (Figs 1 and 2).

\section{Effects of sex hormone deprivation, HFD}

\section{consumption and their combined impact on brain} mitochondrial function

Testosterone deprivation did not induce brain mitochondrial dysfunction in ND-fed male rats (Figs 3A, 4A and 5A), whereas HFD consumption alone and orchiectomy combined with HFD consumption for 12 weeks in male rats caused brain mitochondrial dysfunction at a significant level, demonstrated by an elevation in brain mitochondrial ROS production, brain mitochondrial depolarization and brain mitochondrial swelling (Fig. 3B, 4B, 5B and 3C, 4C, 5C). In contrast, ovariectomized rats which received a ND or sham rats which received HFD for 12 weeks had significantly elevated ROS production, mitochondrial depolarization and mitochondrial swelling (Fig. 3A, 4A, 5A and 3B, 4B, 5B). Interestingly, the estrogen deprivation in obese rats caused brain mitochondrial dysfunction after HFD consumption for 8 weeks (Fig. 3C, 4C and 5C). Analysis of differences between the sexes shows that estrogen deprivation had a higher degree of brain mitochondrial dysfunction when compared with the impact of testosterone deprivation (Fig. 3A, 4A and 5A). When combined with estrogen deprivation, HFD consumption clearly accelerated
A
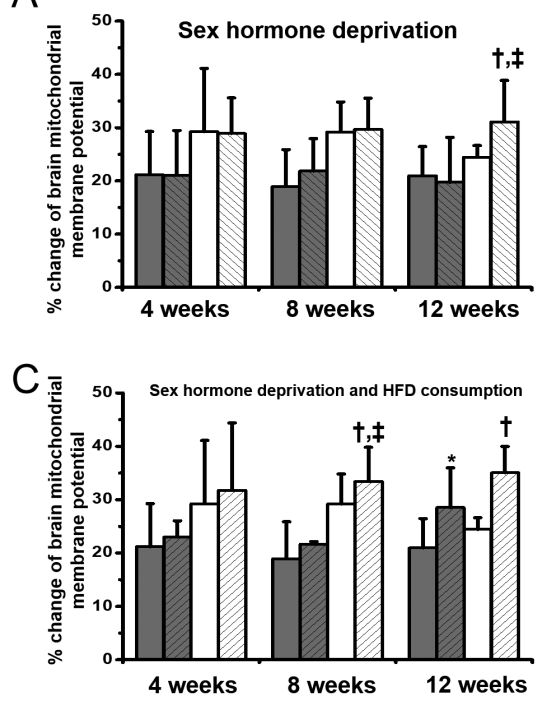
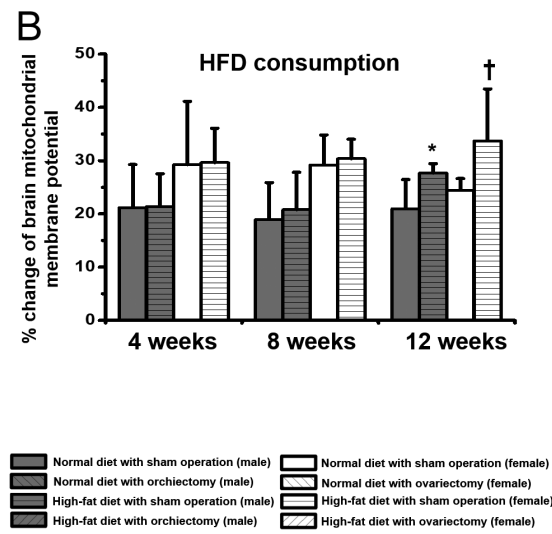

Figure 4

Effects of sex hormone deprivation: (A) HFD consumption; (B) sex hormone deprivation combined with HFD consumption; (C) on the \% change of brain mitochondrial membrane potential. $X$-Axis represents time course of dietary consumption at 4,8 and 12 weeks. $* P<0.05$ vs normal diet-fed male rats with sham operation ${ }^{\dagger} P<0.05$ vs normal diet-fed female rats with sham operation and $¥ P<0.05$ vs male gender with same group. HFD, high-fat diet. 
A
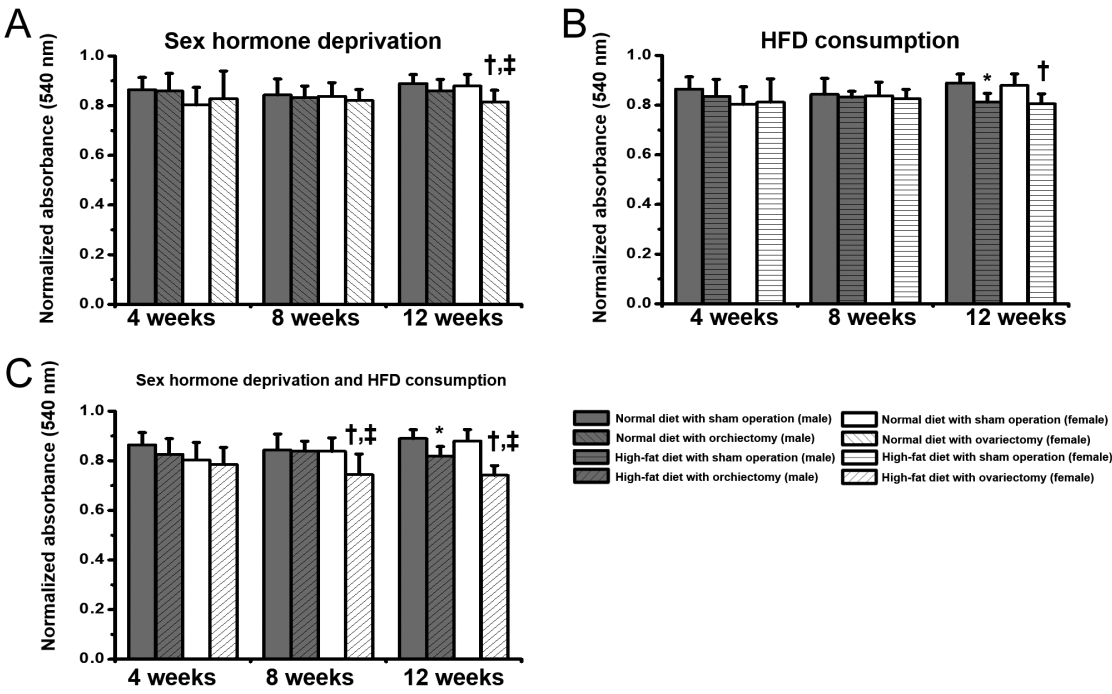

Figure 5

Effects of sex hormone deprivation: (A) HFD consumption; (B) sex hormone deprivation combined with HFD consumption; (C) on the normalized absorbance of brain mitochondria. $X$-Axis represents time course of dietary consumption at 4,8 and 12 weeks. A reduction of normalized absorbance indicates brain mitochondrial swelling. ${ }^{\star} P<0.05$ vs normal diet-fed male rats with sham operation, ${ }^{\dagger} P<0.05$ vs normal diet-fed female rats with sham operation and $¥ P<0.05$ vs male gender with same group. HFD, high-fat diet.

the development of brain mitochondrial dysfunction, compared to testosterone deprivation (Fig. 3C, 4C and 5C). These findings demonstrated that estrogen deprivation had a greater influence on brain mitochondrial function than testosterone deprivation.

\section{Effects of sex hormone deprivation, HFD}

\section{consumption and their combined impact on}

hippocampal synaptic plasticity and insulin-induced LTD

Hippocampal synaptic plasticity is demonstrated by an increase in field excitatory postsynaptic potentials (fEPSPs) or LTP. An impairment of LTP indicated hippocampal dysplasticity. We found that testosterone deprivation accelerated the hippocampal dysplasticity in rats fed with either ND or HFD for 8 weeks (Fig. 6A and C), whereas sham-operated male rats showed this pathological change after HFD consumption for 12 weeks (Fig. 6B). On the other hand, it was observed that the hippocampal dysplasticity in female rats with estrogen deprivation alone, HFD consumption alone and both combined conditions started from 8 weeks of dietary consumption (Fig. 6A, B and C). When compared with the results observed in male rats, the consumption of HFD for 8 weeks was sufficient to trigger a reduction in fEPSPs in female rats rather than the 12 weeks in males (Fig. 6B). According to the results pertinent to insulin-induced LTD, 12 weeks of HFD consumption in male rats that received either a sham operation or testosterone deprivation showed a worsening of insulin-induced LTD as demonstrated by a reduction in the \% decrease of fEPSPs (Fig. 7).

Interestingly, we found that estrogen deprivation in ND-fed rats impaired insulin-induced LTD
A

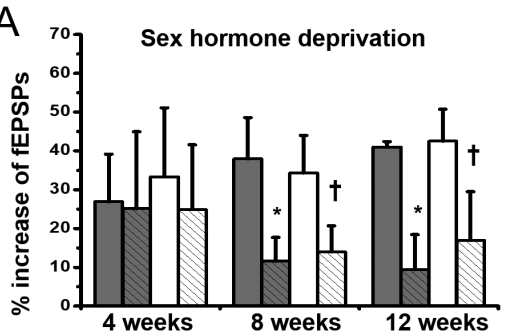

C

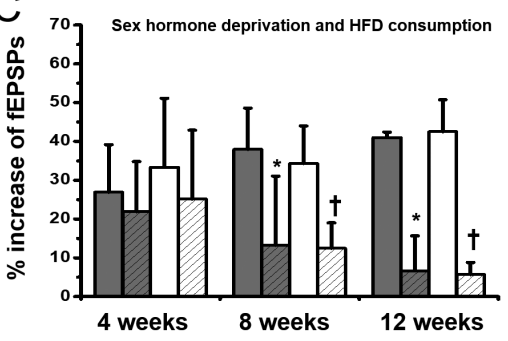

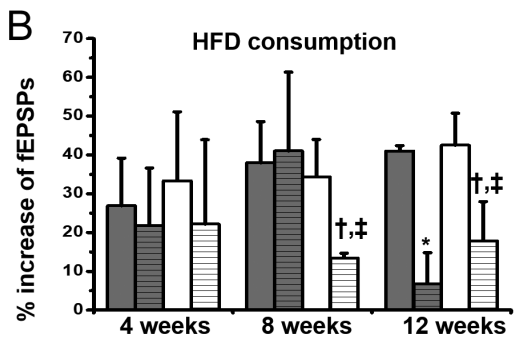

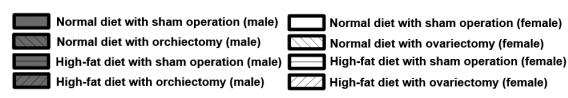

Figure 6

Effects of sex hormone deprivation: (A) HFD consumption; (B) sex hormone deprivation combined with HFD consumption; (C) on the \% increase of fEPSPs. $X$-Axis represents time course of dietary consumption at 4, 8 and 12 weeks. A reduction in $\%$ increase of fEPSP indicated an occurrence of hippocampal dysplasticity. ${ }^{*} P<0.05$ vs normal diet-fed male rats with sham operation, $t P<0.05$ vs normal diet-fed female rats with sham operation and $¥ P<0.05$ vs male gender with same group. fEPSP, field excitatory postsynaptic potential; HFD, high-fat diet. 

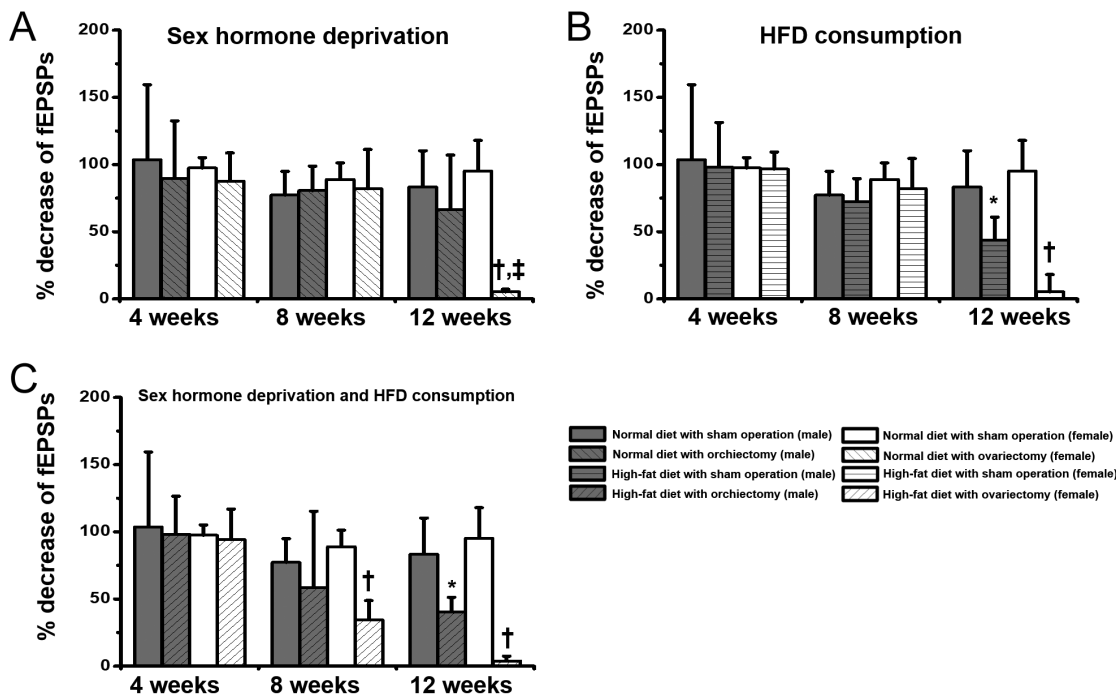

when compared with ND-fed female rats after 12 weeks of dietary consumption (Fig. 7A), whereas HFD consumption for 12 weeks was required to impair this process (Fig. 7B). In addition, a combination of estrogen deprivation with HFD consumption accelerated the impairment of insulin-induced LTD as it was seen at 8 weeks of HFD consumption (Fig. 7C). These findings suggested that consumption of a HFD had more impact on hippocampal synaptic plasticity in female rats than male rats, and that estrogen deprivation lead to a greater aggravation of brain insulin receptor function.

\section{Effects of sex hormone deprivation, HFD consumption and their combined impact on the dendritic spine density and cognitive function}

Orchiectomy could decrease dendritic spine density in rats given with ND for 8 weeks (Fig. 8A), whereas HFD

\begin{abstract}
Figure 7
Effects of sex hormone deprivation: (A) HFD consumption; (B) sex hormone deprivation combined with HFD consumption; (C) on the \% decrease of fEPSPS. $X$-Axis represents time course of dietary consumption at 4, 8 and 12 weeks. A reduction in \% decrease of fEPSP indicates an impairment of insulin-induced long-term depression (LTD). ${ }^{*} P<0.05$ vs normal diet-fed male rats with sham operation, ${ }^{\dagger} P<0.05$ vs normal diet-fed female rats with sham operation and $¥ P<0.05$ vs male gender with same group. fEPSPs, field excitatory postsynaptic potential; HFD, high-fat diet.
\end{abstract}

consumption for 12 weeks led to a significant level of dendritic spine loss in male rats (Fig. 8B). Similarly, obese rats with testosterone deprivation also have a reduction in dendritic spine density following 8 weeks of HFD consumption (Fig. 8C). These findings indicate that dendritic spine loss was accelerated by testosterone deprivation, and that this loss was independent of obesity. Consistent with the findings following orchiectomy, ovariectomized rats with 8 and 12 weeks of normal-diet consumption had a reduction in dendritic spine density (Fig. 8A). In addition, 8 weeks of HFD consumption showed this reduction in rats with the sham operation (Fig. 8B), which occurred faster than in male rats. Surprisingly, estrogen deprivation accelerated the dendritic spine loss at the same time point as in orchiectomized rats (Fig. 8C). However, after HFD consumption for 12 weeks, the dendritic spine density of ovariectomized rats was significantly lower than that
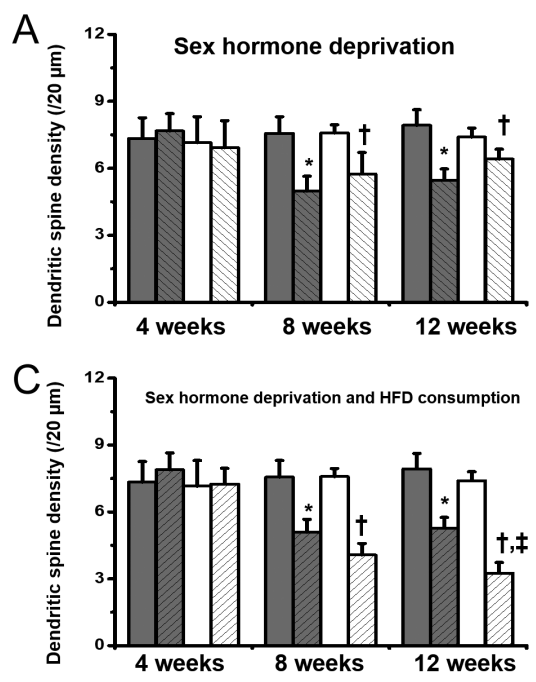
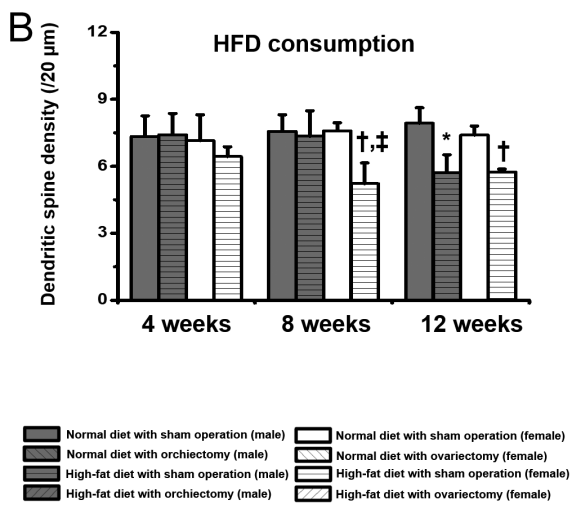

Figure 8

Effects of sex hormone deprivation: (A) HFD consumption; (B) sex hormone deprivation combined with HFD consumption; (C) on the dendritic spine density. $X$-Axis represents time course of dietary consumption at 4, 8 and 12 weeks. ${ }^{*} P<0.05$ vs normal diet-fed male rats with sham operation, ${ }^{\dagger} P<0.05$ vs normal diet-fed female rats with sham operation and $¥ P<0.05$ vs male gender with same group. HFD, high-fat diet. 

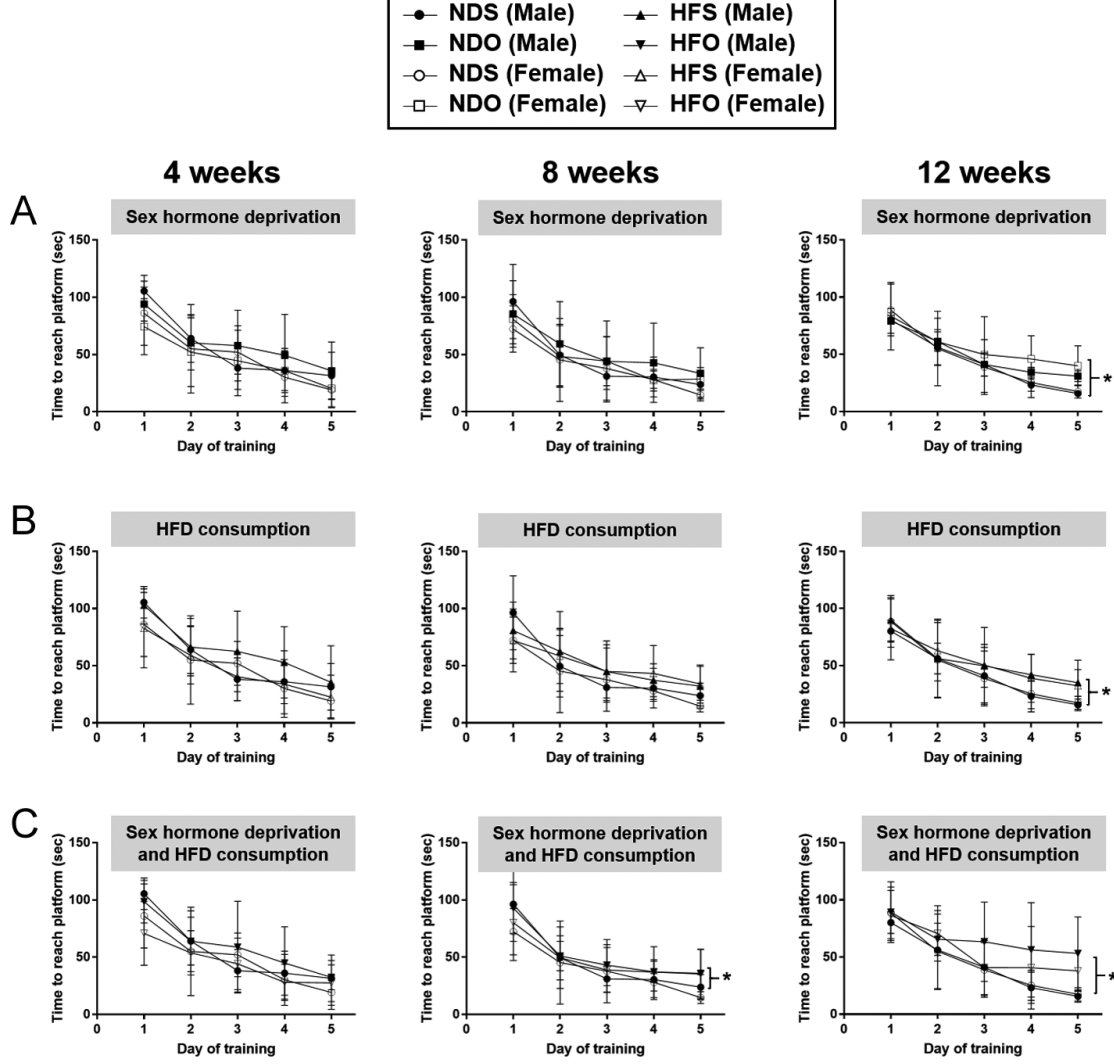

\section{Figure 9}

Effects of sex hormone deprivation: (A) HFD consumption; (B) sex hormone deprivation combined with HFD consumption; (C) at various times of dietary consumption (4, 8 and 12 weeks) on time to reach platform (seconds) which indicates learning function in acquisition test. $\star P<0.05$ vs normal diet-fed male rats with sham operation. HFS, sham-operated HFD-fed rats; ND, normal diet; NDO, ovariectomy-operated ND-fed rats; NDS, sham-operated ND-fed rats. of orchiectomized rats (Fig. 8C). These findings indicate that the reduced dendritic spine density occurred earlier in obese female rats than in obese male rats when the sex hormones are still present, and that estrogen deprivation aggravates this reduction to a greater extent than testosterone deprivation in obese female rats fed with a HFD for 12 weeks.

The results of the acquisition test showed that both testosterone and estrogen deprivation initially impaired learning function of rats fed with either ND or HFD for 12 weeks, as demonstrated by the elevated time to reach the platform compared with the sham-operated rats fed with a ND (Fig. 9C). In addition, HFD consumption in sham-operated rats caused a learning deficit after HFD was given for 12 weeks (Fig. 9F). In addition, rats exposed to the combined conditions of sex hormone deprivation and HFD consumption for 8 weeks in both genders also developed learning impairment (Fig. 9H).
A

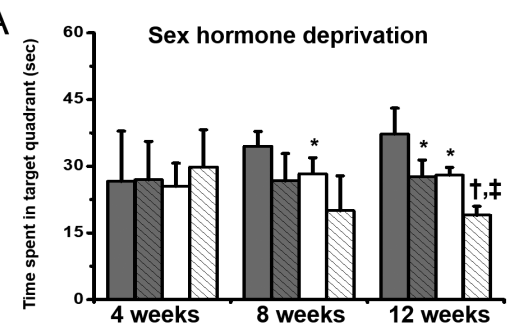

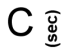

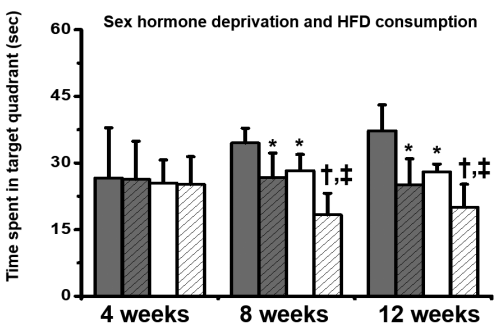

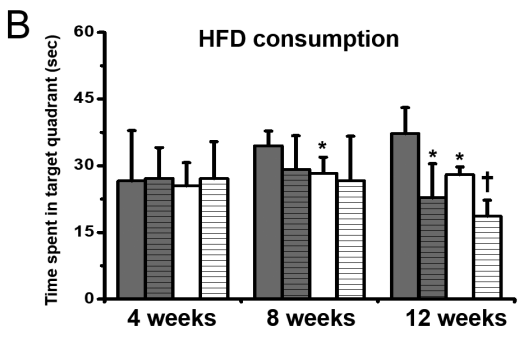

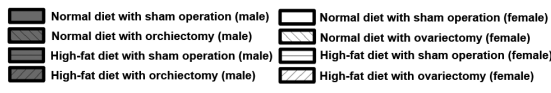

\section{Figure 10}

Effects of sex hormone deprivation: (A) HFD consumption; (B) sex hormone deprivation combined with HFD consumption; (C) on time spent in target quadrant (seconds) which represents the memory function in probe test. $X$-Axis represents time course of dietary consumption at 4,8 and 12 weeks. $* P<0.05$ vs normal diet-fed male rats with sham operation ${ }^{+} P<0.05$ vs normal diet-fed female rats with sham operation and $¥ P<0.05$ vs male gender with same group. HFD, high-fat diet. 
These findings indicated that sex hormone deprivation had equally impaired learning function in both male and female rats.

In contrast, orchiectomized rats and ovariectomized rats developed memory dysfunction as indicated by a reduction in the time spent in the target quadrant (Fig. 10). However, just consumption of HFD for 12 weeks could lead to memory deficit. Additionally, ND-fed female rats which had undergone a sham operation spent a shorter time in the target quadrant compared with male rats (Fig. 10). These findings indicated that memory impairment was accelerated in rats exposed to conditions of sex hormone deprivation.

Lastly, the significant brain parameters from each group were further analyzed to determine the correlation. We found that testosterone-deprived male rats fed with ND has a positive correlation between an increase of fEPSPs and dendritic spine density $(r=1.000, P<0.01)$. Moreover, HFD-fed male rats with sham operation showed the negative correlation in an increase of fEPSPs and brain mitochondrial membrane potential depolarization $(r=-1.000, P<0.01)$. Interestingly, testosterone-deprived male rats fed with HFD showed the negative correlations between (1) an increase of fEPSPs and brain mitochondrial membrane potential depolarization $(r=-1.000, P<0.01)$ and (2) brain insulin-induced LTD and brain mitochondrial ROS production $(r=-1.000, P<0.01)$.

In female rats, estrogen-deprived rats fed with ND demonstrated the association between each parameter including (1) an increase of fEPSPs and dendritic spine density ( $r=0.999, P=0.025)$ and (2) an increase of fEPSPs and brain mitochondrial swelling $(r=-0.999, P=0.035)$. HFD-fed female rats with sham operation also indicated the negative correlation between time to spent in target quadrant from probe test and either brain MDA or brain mitochondrial ROS production $(r=-1.000, P<0.01)$. Additionally, HFD female rats showed the positive association between an increase of fEPSP and brain insulin-induced LTD $(r=-0.968, P=0.032)$, while there was the negative correlation between (1) an increase of fEPSP and brain mitochondrial depolarization $(r=-0.994, P=0.006)$ and (2) time to spend in target quadrant from probe test and brain mitochondrial swelling $(r=-1.000, P<0.01)$.

\section{Discussion}

The major findings from this study are as follows: (1) estrogen deprivation alone and a combination with HFD consumption for 8 weeks induced peripheral insulin resistance and brain dysfunction by increasing oxidative stress, inducing brain mitochondrial dysfunction, impairing insulin-induced LTD, causing hippocampal dysplasticity and dendritic spine reduction, whereas testosterone deprivation alone only caused hippocampal dysplasticity and loss of dendritic spine density, and (2) estrogen deprivation had greater impact on the brain than testosterone deprivation.

In this study, testosterone deprivation in ND-fed rats at any of the time points did not induce peripheral insulin resistance, but when combined with longterm HFD consumption, it triggered peripheral insulin resistance, as shown by elevated levels of body weight, visceral fat, plasma insulin, HOMA index and the disruption of lipid profiles and insulin sensitivity. This suggested that testosterone deprivation alone does not cause the development of peripheral insulin resistance. In contrast, ovariectomy-induced estrogen deprivation significantly influenced this pathological condition in ND-fed rats. In addition, ND-fed female rats had lower levels of peripheral parameters such as body weight, food intake, visceral fats, plasma insulin and HOMA index; however, these rats had higher levels of TC and LDL than that of male rats. These findings might be due to the fact that estrogen plays an important role as a regulator of insulin action and energy balance (Gupte et al. 2015).

With regard to oxidative stress, measured both in the circulation and brain of male rats, stress only occurred in 12-week HFD-fed rats which had undergone a sham operation or orchiectomy, indicating that testosterone deprivation alone does not cause oxidative stress, whereas estrogen deprivation alone, HFD consumption or a combination of estrogen deprivation with HFD consumption led to the induction of oxidative stress. Furthermore, ND-fed female rats also had greater level of oxidative stress when compared with male rats. Taken together, these findings suggest that female rats are more susceptible to oxidative stress when compared to males. In support of this finding, estrogen has been reported to regulate antioxidant genes (Baltgalvis et al. 2010) and modulate antioxidant enzymes in circulation (Bellanti et al. 2013). Thus, the deprivation of estrogen results in the increased severity of oxidative stress when compared to testosterone deprivation as observed in the present study.

Interestingly, brain mitochondrial dysfunction was not observed in testosterone-deprived rats fed with a $\mathrm{ND}$, whereas HFD-fed rats with or without testosterone 
deprivation had brain mitochondrial dysfunction as shown by elevated mitochondrial ROS production, mitochondrial membrane potential change and mitochondrial swelling, suggesting that brain mitochondrial dysfunction in males occurred in a testosterone-independent manner. On the contrary, in female rats fed with either ND or HFD, estrogen deprivation was sufficient to cause brain mitochondrial dysfunction. This supports the finding that estrogen is required for regulating mitochondrial structure and function, particularly in the brain (Klinge 2008, Arnold et al. 2012).

As mentioned above, estrogen plays a significant role in insulin action. Consistent with this finding, the present study also shows that estrogen deprivation resulted in impaired insulin-induced LTD, which represents brain insulin action, whereas testosterone deprivation alone had not affected the insulin-induced LTD. The impaired LTD during estrogen deprivation might be due to the loss of the insulin signaling response from estrogen which can activate insulin receptor substrate-1 (IRS-1) after binding with the estrogen receptors in the brains (Gonzalez et al. 2008). Moreover, estrogen deprivation combined with chronic consumption of HFD accelerated the impairment of brain insulin action since this combined condition decreased LTD after 8 weeks of HFD consumption, while estrogen deprivation alone only disrupted this process in 12 weeks ND-fed rats. This suggests that estrogen plays a dominant role in brain insulin action compared with testosterone.

Hippocampal synaptic plasticity, a process fundamental to learning and memory, is impaired in both testosterone- and estrogen-deprived rats. Moreover, dendritic spine density was also reduced in these two groups. As a result, these alterations led to cognitive dysfunction, as demonstrated in Morris water maze results. Under estrogen-deprived conditions, these impairments could be explained by the induction of oxidative stress that results in a reduction in synaptic plasticity, as shown in previous reports (Massaad \& Klann 2011, Pratchayasakul et al. 2015). On the other hand, because the oxidative stress of orchiectomized rats did not increase, further investigation is needed to clarify whether testosterone deprivation induced hippocampal synaptic dysplasticity, dendritic spine reduction and eventually cognitive impairment.

Interestingly, we found that although estrogen deprivation accelerated peripheral insulin resistance and brain dysfunction, but not in testosterone deprivation, the induction of cognitive impairment occurred at the same time point in each group. The possible explanations for these findings are: (1) estrogen and testosterone play an important role for cognitive function (Li \& Singh 2014, Hamson et al. 2016). The cognitive impairment following the deprivation of both sex hormones might be at the maximal level, so other factors are no longer causing further impairment; and (2) the acceleration to cause further cognitive impairment in the combined hormonal deprivation and obesity may require longer time of HFD consumption.

Considering the correlations among all parameters, we found that under sex hormone deprivation alone, there was a correlation within hippocampal dysplasticity, brain mitochondrial dysfunction and dendritic spine loss in ovariectomized rats, whereas there was an association between hippocampal dysplasticity and dendritic spine in orchiectomized rats. These findings suggested that hippocampal dysplasticity and the loss of dendritic spine under testosterone deprivation might be the major factors in the cognitive impairment, while estrogen deprivation induced cognitive impairment through the several mechanisms previously mentioned. In contrast, we found that HFD consumption alone in both male and female rats showed a significant correlation in oxidative stress, brain mitochondrial dysfunction, impaired brain insulininduced LTD, hippocampal dysplasticity, dendritic spine loss and cognitive impairment, suggesting that long-term HFD consumption led to cognitive impairment in both genders. Moreover, there was a correlation regarding these parameters under a combination of HFD consumption and sex hormone deprivation of both males and females. Taken together, HFD consumption might have a higher impact in the induction of cognitive impairment in male rats, compared with testosterone deprivation. In contrast, both HFD consumption and estrogen deprivation had equal effects in inducing cognitive impairment in female rats. These findings suggested a more dominant role of estrogen in preserving brain function than testosterone.

\section{Conclusion}

This study has demonstrated that estrogen deprivation has much greater impact on the brain than does testosterone deprivation. These findings suggest the possibility that menopausal women have more severe brain pathologies after hormone deprivation in comparison to andropause men. In addition, this indicates the importance of estrogen in the preservation of brain function, and that estrogenreplacement therapy would be a potential strategy to slow cognitive decline in menopausal women. 


\section{Study limitations}

Although an increase in both body weight and visceral fat were observed in the present study, the other direct indicators for obesity such as BMI are still required. In addition, the assessment of oxidative damage to proteins or DNA is needed to investigate whether sex hormone deprivation, HFD consumption or a combination of these conditions induce oxidative stress in blood circulation and the brain.

Furthermore, a further longitudinal study across aging should be conducted to show the effects of gradual reduction in sex hormones on the same parameters of the present study.

\section{Supplementary data}

This is linked to the online version of the paper at https://doi.org/10.1530/ JOE-18-0552.

\section{Declaration of interest}

The authors declare that there is no conflict of interest that could be perceived as prejudicing the impartiality of the research reported.

\section{Funding}

This work was supported by the Thailand Research Fund: Senior Research Scholar RTA6080003 (S C C), MRG6080226 (I S), RSA6180071 (W P) and the NSTDA Research Chair Grant from the National Science and Technology Development Agency Thailand (N C), and Chiang Mai University Center of Excellence Award (N C).

\section{References}

Arnold S, Victor MB \& Beyer C 2012 Estrogen and the regulation of mitochondrial structure and function in the brain. Journal of Steroid Biochemistry and Molecular Biology 131 2-9. (https://doi.org/10.1016/j. jsbmb.2012.01.012)

Baltgalvis KA, Greising SM, Warren GL \& Lowe DA 2010 Estrogen regulates estrogen receptors and antioxidant gene expression in mouse skeletal muscle. PLOS ONE 5 e10164. (https://doi.org/10.1371/ journal.pone.0010164)

Bellanti F, Matteo M, Rollo T, De Rosario F, Greco P, Vendemiale G \& Serviddio G 2013 Sex hormones modulate circulating antioxidant enzymes: impact of estrogen therapy. Redox Biology 1 340-346. (https://doi.org/10.1016/j.redox.2013.05.003)

Chattipakorn SC \& McMahon LL 2002 Pharmacological characterization of glycine-gated chloride currents recorded in rat hippocampal slices. Journal of Neurophysiology 87 1515-1525. (https://doi.org/10.1152/ jn.00365.2001)

Daniel JM, Fader AJ, Spencer AL \& Dohanich GP 1997 Estrogen enhances performance of female rats during acquisition of a radial arm maze. Hormones and Behavior 32 217-225. (https://doi.org/10.1006/ hbeh.1997.1433)
Ginsberg HN 2002 Risk factors common to insulin resistance and atherosclerosis explain why diabetes is a cardiovascular disease. American Journal of Managed Care $\mathbf{3}$ (Supplement) 5.

Gonzalez C, Diaz F \& Alonso A 2008 Neuroprotective effects of estrogens: cross-talk between estrogen and intracellular insulin signalling. Infectious Disorders Drug Targets 8 65-67. (https://doi. org/10.2174/187152608784139659)

Grossmann M, Thomas MC, Panagiotopoulos S, Sharpe K, Macisaac RJ, Clarke S, Zajac JD \& Jerums G 2008 Low testosterone levels are common and associated with insulin resistance in men with diabetes. Journal of Clinical Endocrinology and Metabolism 93 1834-1840. (https://doi.org/10.1210/jc.2007-2177)

Gupte AA, Pownall HJ \& Hamilton DJ 2015 Estrogen: an emerging regulator of insulin action and mitochondrial function. Journal of Diabetes Research 2015 916585. (https://doi. org/10.1155/2015/916585)

Hamson DK, Roes MM \& Galea LA 2016 Sex hormones and cognition: neuroendocrine influences on memory and learning. Comprehensive Physiology 6 1295-1337. (https://doi.org/10.1002/cphy.c150031)

Hogervorst E, Williams J, Budge M, Barnetson L, Combrinck M \& Smith AD 2001 Serum total testosterone is lower in men with Alzheimer's disease. Neuroendocrinology Letters 22 163-168.

Kenny AM, Fabregas G, Song C, Biskup B \& Bellantonio S 2004 Effects of testosterone on behavior, depression, and cognitive function in older men with mild cognitive loss. Journals of Gerontology: Series A, Biological Sciences and Medical Sciences 59 75-78. (https://doi. org/10.1093/gerona/59.1.M75)

Klinge CM 2008 Estrogenic control of mitochondrial function and biogenesis. Journal of Cellular Biochemistry 105 1342-1351. (https:// doi.org/10.1002/jcb.21936)

Li R \& Singh M 2014 Sex differences in cognitive impairment and Alzheimer's disease. Frontiers in Neuroendocrinology 35 385-403. (https://doi.org/10.1016/j.yfrne.2014.01.002)

Luine VN, Richards ST, Wu VY \& Beck KD 1998 Estradiol enhances learning and memory in a spatial memory task and effects levels of monoaminergic neurotransmitters. Hormones and Behavior 34 149-162. (https://doi.org/10.1006/hbeh.1998.1473)

Maciejczyk M, Zebrowska E, Zalewska A \& Chabowski A 2018 Redox balance, antioxidant defense, and oxidative damage in the hypothalamus and cerebral cortex of rats with high fat diet-induced insulin resistance. Oxidative Medicine and Cellular Longevity 2018 6940515. (https://doi.org/10.1155/2018/6940515)

Massaad CA \& Klann E 2011 Reactive oxygen species in the regulation of synaptic plasticity and memory. Antioxidants and Redox Signaling 14 2013-2054. (https://doi.org/10.1089/ars.2010.3208)

Naghdi N, Majlessi N \& Bozorgmehr T 2005 The effect of intrahippocampal injection of testosterone enanthate (an androgen receptor agonist) and anisomycin (protein synthesis inhibitor) on spatial learning and memory in adult, male rats. Behavioural Brain Research 156 263-268. (https://doi.org/10.1016/j.bbr.2004.05.032)

Pintana H, Apaijai N, Pratchayasakul W, Chattipakorn N \& Chattipakorn SC 2012 Effects of metformin on learning and memory behaviors and brain mitochondrial functions in high fat diet induced insulin resistant rats. Life Sciences 91 409-414. (https://doi. org/10.1016/j.lfs.2012.08.017)

Pintana H, Apaijai N, Chattipakorn N \& Chattipakorn SC 2013 DPP-4 inhibitors improve cognition and brain mitochondrial function of insulin-resistant rats. Journal of Endocrinology 218 1-11. (https://doi. org/10.1530/JOE-12-0521)

Pintana H, Chattipakorn N \& Chattipakorn S 2015a Testosterone deficiency, insulin-resistant obesity and cognitive function. Metabolic Brain Disease 30 853-876. (https://doi.org/10.1007/s11011-015-9655-3)

Pintana H, Pongkan W, Pratchayasakul W, Chattipakorn N \& Chattipakorn SC $2015 b$ Dipeptidyl peptidase 4 inhibitor improves brain insulin sensitivity, but fails to prevent cognitive impairment https://joe.bioscientifica.com https://doi.org/10.1530/JOE-18-0552
(C) 2019 Society for Endocrinology Published by Bioscientifica Ltd. Printed in Great Britain 
in orchiectomy obese rats. Journal of Endocrinology 226 M1-M11. (https://doi.org/10.1530/JOE-15-0099)

Pintana H, Pratchayasakul W, Sa-nguanmoo P, Pongkan W, Tawinvisan R, Chattipakorn N\& Chattipakorn SC 2016 Testosterone deprivation has neither additive nor synergistic effects with obesity on the cognitive impairment in orchiectomized and/or obese male rats. Metabolism 65 54-67. (https://doi.org/10.1016/j.metabol.2015.10.015)

Pipatpiboon N, Pratchayasakul W, Chattipakorn N \& Chattipakorn SC 2012 PPARgamma agonist improves neuronal insulin receptor function in hippocampus and brain mitochondria function in rats with insulin resistance induced by long term high-fat diets. Endocrinology 153 329-338. (https://doi.org/10.1210/en.2011-1502)

Pipatpiboon N, Pintana H, Pratchayasakul W, Chattipakorn N \& Chattipakorn SC 2013 DPP4-inhibitor improves neuronal insulin receptor function, brain mitochondrial function and cognitive function in rats with insulin resistance induced by high-fat diet consumption. European Journal of Neuroscience 37 839-849. (https:// doi.org/10.1111/ejn.12088)

Pratchayasakul W, Kerdphoo S, Petsophonsakul P, Pongchaidecha A, Chattipakorn N\& Chattipakorn SC 2011 Effects of high-fat diet on insulin receptor function in rat hippocampus and the level of neuronal corticosterone. Life Sciences 88 619-627. (https://doi. $\operatorname{org} / 10.1016 / j . l f s .2011 .02 .003)$

Pratchayasakul W, Sa-Nguanmoo P, Sivasinprasasn S, Pintana H, Tawinvisan R, Sripetchwandee J, Kumfu S, Chattipakorn N \& Chattipakorn SC 2015 Obesity accelerates cognitive decline by aggravating mitochondrial dysfunction, insulin resistance and synaptic dysfunction under estrogen-deprived conditions. Hormones and Behavior 72 68-77. (https://doi.org/10.1016/j.yhbeh.2015.04.023)
Sakata K, Tokue A \& Kawai N 2000 Altered synaptic transmission in the hippocampus of the castrated male mouse is reversed by testosterone replacement. Journal of Urology 163 1333-1338. (https://doi. org/10.1016/S0022-5347(05)67773-7)

Sandstrom NJ, Kim JH \& Wasserman MA 2006 Testosterone modulates performance on a spatial working memory task in male rats. Hormones and Behavior 50 18-26. (https://doi.org/10.1016/j. yhbeh.2005.09.008)

Schiavi RC \& White D 1976 Androgens and male sexual function: a review of human studies. Journal of Sex and Marital Therapy 2 214-228. (https://doi.org/10.1080/00926237608405324)

Sripetchwandee J, Sanit J, Chattipakorn N \& Chattipakorn SC 2013 Mitochondrial calcium uniporter blocker effectively prevents brain mitochondrial dysfunction caused by iron overload. Life Sciences 92 298-304. (https://doi.org/10.1016/j.lfs.2013.01.004)

Taylor R 2012 Insulin resistance and type 2 diabetes. Diabetes 61 778-779. (https://doi.org/10.2337/db12-0073)

Vorhees CV \& Williams MT 2006 Morris water maze: procedures for assessing spatial and related forms of learning and memory. Nature Protocols 1 848-858. (https://doi.org/10.1038/nprot.2006.116)

Watts AS, Loskutova N, Burns JM \& Johnson DK 2013 Metabolic syndrome and cognitive decline in early Alzheimer's disease and healthy older adults. Journal of Alzheimer's Disease 35 253-265. (https://doi.org/10.3233/JAD-121168)

Xia F, Xu X, Zhai H, Meng Y, Zhang H, Du S, Xu H, Wu H \& Lu Y 2013 Castration-induced testosterone deficiency increases fasting glucose associated with hepatic and extra-hepatic insulin resistance in adult male rats. Reproductive Biology and Endocrinology 11 106. (https://doi. org/10.1186/1477-7827-11-106)

Received in final form 13 January 2019

Accepted 28 January 2019

Accepted Preprint published online 28 January 2019
(C) 2019 Society for Endocrinology Published by Bioscientifica Ltd. 